\title{
Simulations of dislocation density in silicon carbide crystals grown by the PVT-method
}

\author{
Qi-Sheng Chen ${ }^{\mathrm{a}, \mathrm{b}, \mathrm{c}, *}$, Peng Zhu ${ }^{\mathrm{a}, \mathrm{c}}$, Meng $\mathrm{He}^{\mathrm{a}, \mathrm{c}}$ \\ ${ }^{\text {a }}$ Key Laboratory of Microgravity, Institute of Mechanics, Chinese Academy of Sciences, Beijing 100190, China \\ ${ }^{\mathrm{b}}$ Center of Materials Science and Optoelectronics Engineering, University of Chinese Academy of Sciences, Beijing 100049, China \\ ${ }^{\mathrm{c}}$ School of Engineering Science, University of Chinese Academy of Sciences, Beijing 100190, China
}

\section{A R T I C L E I N F O}

Communicated by Min Lu

Keywords:

A1. Computer simulation

A1. Defects

A1. Heat transfer

A1. Stresses

A2. Growth from vapor

B2. Semiconducting silicon compounds

\begin{abstract}
A B S T R A C T
The Alexander-Haasen (AH) model has been applied to analyze the plastic deformation and dislocation generation during the crystal growth process of $4 \mathrm{H}-\mathrm{SiC}$ (silicon carbide). Plastic parameters are obtained by fitting the predicted curves to the experimental data on the plastic deformation of $\alpha$-SiC crystals under uniaxial compression. The relationship between the activity energy $(Q)$ and stress exponent $(n)$ is considered when using the AH model. This relationship explicitly represents two deformation mechanisms around the critical temperature. The ratio of the activity energy and stress exponent, $Q / n$, equals $0.3 \mathrm{eV}$ when the temperature is below the transition temperature, and $1.3 \mathrm{eV}$ when the temperature is above the transition temperature. Then, the model is used to predict the dislocation density and thermal stresses in the crystals. The largest dislocation density is found to occur near the graphite/SiC interface, and the dislocation density gradually decreases with the thickness of the ingot.
\end{abstract}

\section{Introduction}

Silicon carbide $(\mathrm{SiC})$ is an advanced semiconducting material which is generally produced by the physical vapor transport (PVT) method. The growth process involves many important physico-chemical phenomena, such as the electromagnetic induction heating, coupled heat and mass transfer, phase transformation, chemical reactions, etc. [1]. For those physico-chemical problems, researchers have studied the crystal growth mechanism by numerical simulations and experiments [2-5]. Based on the phase diagram of SiC [6], the SiC crystals can be grown in a large temperature range, which increases the difficulties to produce the perfect crystal bulks through the system design and process optimization. As a result, the important and difficult point is how to obtain a specific temperature field where the radial and axial temperature gradients at the crystallization surface become suitable for crystal enlargement [7-8]. Due to the non-uniformity of temperature field in the crystal growth system, thermal stresses are induced, which can cause the plastic deformation, dislocation generation and multiplication during the growth process. Many progresses have been made in reducing the crystal defects, such as the micropipe defects and angle boundaries [9]. However, the formation and expansion of stacking faults in the basal plane causes degradation of the bipolar $4 \mathrm{H}$ - and $6 \mathrm{H}-$ $\mathrm{SiC}$ devices, whose edges are identified to be Shockley partial dislocations [10]. Thus, the properties, generation and multiplication of dislocations in the basal plane have become the focus of research for reducing its density in the bulk crystals grown by the PVT method.

The first work on the plastic deformation of silicon carbide was done by Fujita et al. [11] in 1987. They found that some specimens only result in apparent elastic deformation before buckling fracture and some specimens deform plastically, when compression tests are performed with specimens of $6 \mathrm{H}$-SiC crystals with different orientations at temperatures between $1100{ }^{\circ} \mathrm{C}$ and $1800{ }^{\circ} \mathrm{C}$. They also found that the resolved shear stress is a function of temperature. Later, Samant and Pirouz [12] performed compression experiments with $6 \mathrm{H}$-SiC crystals at temperatures from $700{ }^{\circ} \mathrm{C}$ to $1300{ }^{\circ} \mathrm{C}$, and observed a change in the deformation mechanism at the temperature around $1100{ }^{\circ} \mathrm{C}$. TEM observations of the dislocation microstructure of deformed specimens at temperatures above $1100{ }^{\circ} \mathrm{C}$ show the presence of perfect dislocations, which consist of leading and trailing partials. Below this transition temperature, only one of the partial basal dislocations was observed, together with the existence of large areas of stacking faults. Similar experimental phenomena in the properties of the micro-structure of dislocations were found in the compression experiments on $4 \mathrm{H}-\mathrm{SiC}$ by Demenet et al [13]. Uniaxial compression tests with large strains near $10 \%$ performed by Lara et al. [14] on the plastic deformation of $4 \mathrm{H}-\mathrm{SiC}$ samples also show the two types of deformation mechanisms at

\footnotetext{
* Corresponding author at: Key Laboratory of Microgravity, Institute of Mechanics, Chinese Academy of Sciences, Beijing 100190, China.

E-mail address: qschen@imech.ac.cn (Q.-S. Chen).
} 
temperatures between $800{ }^{\circ} \mathrm{C}$ and $1300{ }^{\circ} \mathrm{C}$. They found the transition temperature is about $1030{ }^{\circ} \mathrm{C}$. Those works help us to understand the plastic deformation behaviors of silicon carbide in different temperature ranges. However, the real deformation of silicon carbide occurring in the growth process is very difficult to be detected since the growth temperature is usually above $2000{ }^{\circ} \mathrm{C}[6]$.

To overcome the real observation obstacles, we applied the Alexander-Haasen (AH) model to describe the plastic deformation behaviors of $\mathrm{SiC}$ crystals. The $\mathrm{AH}$ model is time-dependent for the plastic deformation and dislocation multiplication based on the plastic strain rate and dislocation multiplication rate equations. Owing to its extraordinary ability to model plastic deformations of elemental and III-V compound crystals [15-17], the AH model has been increasingly used to simulate the plastic deformation and dislocation generation in the IVIV compound crystals [18-19]. Gao and Kakimoto [19] compared the predicted results with the experimental data from Ref. [14], but they didn't consider the two different deformation mechanisms in their studies.

In this paper, plastic parameters are calculated through fitting the predicted results to the uniaxial compression experimental data by considering the two crystal deformation mechanisms. Then the $\mathrm{AH}$ model is used to analyze the plastic deformation and dislocation generation in SiC crystals.

\section{Mathematical model}

\subsection{1-D plastic model}

The mean velocity $\bar{v}$ of dislocations in a covalent crystal is described as a function of the effective stress $\tau_{\text {eff }}$ by the following equation,

$\bar{v}=v_{0}\left(\frac{\tau_{\text {eff }}}{\tau_{0}}\right)^{n} \exp \left(-Q / k_{\mathrm{B}} T\right)$

where $v_{0}$ is a constant, $\mathrm{n}$ is the stress exponent, the symbol $\mathrm{Q}$ is the activation energy for the dislocation glide independent of the temperature and stress, the Boltzmann's constant $k_{\mathrm{B}}=8.617 \times 10^{-5} \mathrm{eV} \mathrm{K}^{-1}$, the reference stress $\tau_{0}=1 \mathrm{MPa}$, and $\mathrm{T}$ is temperature in Kelvin. In Eq. (1), the effective stress $\tau_{\text {eff }}$ is given by

$\tau_{\text {eff }}=\left\langle\tau_{\text {res }}-D \sqrt{N_{\mathrm{m}}}\right\rangle$

where $\tau_{\text {res }}$ denotes the resolved shear stress acting on the slip plane, $D$ is the material hardening factor, and $\langle x\rangle=x$ if $x>0$, and 0 otherwise. It should be noted that the term $D \sqrt{N_{\mathrm{m}}}$ denotes the internal long-range elastic stress generated by mobile dislocations, meanwhile the shortrange interactions are neglected [20].

The dislocation density affects the mechanical deformation behavior of silicon carbide crystals even at low strain rates $\left(\dot{\varepsilon}=10^{-5} \mathrm{~s}^{-1}\right)$. This effect becomes more obvious at temperatures above $800{ }^{\circ} \mathrm{C}$. Plastic deformation of $4 \mathrm{H}-\mathrm{SiC}$ is assumed to be proceeded solely by the motion and multiplication of dislocations on their slip planes. The total dislocations density $N$ on a basal slip system is assumed to be composed of the density of mobile segments carrying plastic flow, $N_{\mathrm{m}}$, and the density of immobile ones trapped in other defects, $N_{\mathrm{i}}$. Here $N_{\mathrm{i}}$ is excluded in our discussion. Due to the dislocation glide mechanism, the gap between the macroscopic plastic strain rate $\dot{\gamma}_{\mathrm{p}}$ and microstructural dislocation density $N_{\mathrm{m}}$ is bridged by the Orowan's law [21],

$\dot{\gamma}_{\mathrm{p}}=N_{\mathrm{m}} b \bar{v}$

where $b$ is the magnitude of the Burgers vector of dislocations, $\bar{v}$ is the mean velocity of moving dislocations in the basal slip system. Combining Eqs. (1)-(3), the following equation is obtained,

$\dot{\gamma}_{\mathrm{p}}=b v_{0} N_{\mathrm{m}}\left\langle\frac{\tau_{\text {res }}-D \sqrt{N_{\mathrm{m}}}}{\tau_{0}}\right\rangle^{n} \exp \left(-Q / k_{\mathrm{B}} T\right)$

The plastic shear deformation is caused by a shear stress $\tau_{\text {res }}$ resolved onto the shear plane, resulting from the externally applied stress $\sigma$ according to the Schmid's law [22],

$\tau_{\text {res }}=m_{\mathrm{s}} \sigma$

where $m_{\mathrm{s}}$ is the orientation factor (also called the stress Schmid factor), which is set to be 0.5 according to the uniaxial compression test [14]. The shear strain in the slip plane obtained by strain transform is described by the following expression,

$\gamma=(1+\nu) \varepsilon$

where $\varepsilon$ is the compression strain, and $\nu$ is the Poisson ratio which equals to 0.16 [23].

The shear stress $\tau_{\text {res }}$ acted in the slip plane is proportional to the elastic shear strain, $\gamma_{\mathrm{e}}$,

$\tau_{\text {res }}=\xi \gamma_{\mathrm{e}}=\xi\left(\gamma-\gamma_{\mathrm{p}}\right)$

where $\xi$ is the shear modulus of the whole experimental system, and $\gamma$ is the total shear strain in the slip plane. Applying derivation of Eq. (7) with respect to the compression strain, the following equation is obtained,

$\frac{d \tau_{\text {res }}}{d \varepsilon}=\xi \frac{d\left(\gamma-\gamma_{\mathrm{p}}\right)}{d \varepsilon}$

Combining Eqs. (4)-(6) and (8), the following equation is obtained,

$m_{s} \frac{d \sigma}{d \varepsilon}=\xi\left[(1+\nu)-\frac{b v_{0} N_{m}}{d \varepsilon / d t}\left\langle\frac{\tau_{\text {res }}-D \sqrt{N_{m}}}{\tau_{0}}\right\rangle^{n} \exp \left(-Q / k_{B} T\right)\right]$

When studying the stress-strain curve of crystals in compression experiments, the derivation of compression strain $d \varepsilon$ equals $\dot{\varepsilon} d t$, where $\dot{\varepsilon}$ is the strain rate depending on the speed of the experiment machine cross-head, and $t$ is the time.

The mobile dislocation density rate is described by the following expression,

$\dot{N}_{\mathrm{m}}=\frac{K}{b}\left(\frac{\tau_{\text {eff }}}{\tau_{0}}\right)^{\lambda} \dot{\gamma}_{\mathrm{p}}$

where $K$ is a multiplication constant at the given loading conditions, and $\lambda$ is a material factor characterizing the dislocation multiplication effect. Similarly, substituting Eqs. (2) and (4) into Eq. (10), the mobile dislocation density rate is obtained,

$\dot{N}_{\mathrm{m}}=K v_{0} N_{\mathrm{m}}\left\langle\frac{m_{\mathrm{S}} \sigma-D \sqrt{N_{\mathrm{m}}}}{\tau_{0}}\right\rangle^{n+\lambda} \exp \left(-Q / k_{\mathrm{B}} T\right)$

\subsection{2-D plastic model}

Thermal stresses caused by the non-uniform temperature field in the growth system can induce plastic deformation and dislocation generation. In the 2-D model, the normal stress components, $\sigma_{\mathrm{rr}} ., \sigma_{\varphi \varphi}, \sigma_{\mathrm{zz}}$, and the shear stress component, $\tau_{\mathrm{rz}}$, are considered. These stress components are used to calculate the second invariant of the deviatoric stress $J_{2}$,

$J_{2}=1 / 6\left[\left(\sigma_{\mathrm{rr}}-\sigma_{\varphi \varphi}\right)^{2}+\left(\sigma_{\mathrm{rr}}-\sigma_{\mathrm{zz}}\right)^{2}+\left(\sigma_{\varphi \varphi}-\sigma_{\mathrm{zz}}\right)^{2}\right]+\tau_{\mathrm{rz}}^{2}$

The effective stress in this case is obtained by substituting $\sqrt{J_{2}}$ for $\tau_{\text {res }}$ in Eq. (2), and the plastic strain components are postulated to be in the following expression [24],

$\frac{d}{d t}\left[\begin{array}{c}\varepsilon_{\mathrm{rr}, \mathrm{p}} \\ \varepsilon_{\varphi \varphi, \mathrm{p}} \\ \varepsilon_{\mathrm{zz}, \mathrm{p}} \\ \gamma_{\mathrm{rz}, \mathrm{p}}\end{array}\right]=\frac{b v_{0} N_{\mathrm{m}}}{2 \sqrt{J_{2}}}\left\langle\frac{\sqrt{J_{2}}-D \sqrt{N_{\mathrm{m}}}}{\tau_{0}}\right\rangle^{n} \exp \left(-\frac{Q}{k_{\mathrm{B}} T}\right)\left[\begin{array}{c}\sigma_{\mathrm{rr}}-\sigma_{\mathrm{m}} \\ \sigma_{\varphi \varphi}-\sigma_{\mathrm{m}} \\ \sigma_{\mathrm{zz}}-\sigma_{\mathrm{m}} \\ \tau_{\mathrm{rz}}\end{array}\right]$

where the spherical stress, $\sigma_{\mathrm{m}}=1 / 3\left(\sigma_{\mathrm{rr}}+\sigma_{\varphi \varphi}+\sigma_{\mathrm{zz}}\right)$. The dislocation density is obtained by substituting $\sqrt{J_{2}}$ for $m_{\mathrm{S}} \sigma$ in Eq. (11). Then the constitutive relation for the thermo-elastic anisotropic body is assumed 
to be,

$\frac{d}{d t}\left[\begin{array}{c}\sigma_{\mathrm{rr}} \\ \sigma_{\varphi \varphi} \\ \sigma_{\mathrm{zz}} \\ \tau_{\mathrm{rz}}\end{array}\right]=\left[\begin{array}{cccc}c_{11} & c_{12} & c_{13} & 0 \\ c_{12} & c_{22} & c_{23} & 0 \\ c_{13} & c_{23} & c_{33} & 0 \\ 0 & 0 & 0 & c_{44}\end{array}\right] \cdot \frac{d}{d t}\left[\begin{array}{c}\varepsilon_{\mathrm{rr}}-\varepsilon_{\mathrm{rr}, \mathrm{p}}-\alpha_{r}\left(T-T_{\mathrm{ref}}\right) \\ \varepsilon_{\varphi \varphi}-\varepsilon_{\varphi \varphi, \mathrm{p}}-\alpha_{\varphi}\left(T-T_{\mathrm{ref}}\right) \\ \varepsilon_{\mathrm{zz}}-\varepsilon_{\mathrm{zz}, \mathrm{p}}-\alpha_{\mathrm{z}}\left(T-T_{\mathrm{ref}}\right) \\ \gamma_{\mathrm{rz}}-\gamma_{\mathrm{rz}, \mathrm{p}}\end{array}\right]$

where $T_{\text {ref }}$ is the reference temperature, $\varepsilon_{\mathrm{rr}}=\frac{\partial u}{\partial r}, \varepsilon_{\varphi \varphi}=\frac{u}{r}, \varepsilon_{\mathrm{zz}}=\frac{\partial w}{\partial z}$, $\gamma_{\mathrm{rz}}=\frac{\partial u}{\partial z}+\frac{\partial w}{\partial r}$ are the strain components, $\varepsilon_{\mathrm{rr}, \mathrm{p}}, \varepsilon_{\varphi \varphi, \mathrm{p}}, \varepsilon_{\mathrm{zz}, \mathrm{p}}, \gamma_{\mathrm{rz}, \mathrm{p}}$ denote the plastic strains, $\mathrm{u}$ and $\mathrm{w}$ are the displacements in the radial and axial directions, respectively, $c_{\mathrm{ij}}(i, j=1,2,3)$ and $c_{44}$ are the elastic constants, and $\alpha_{\mathrm{r}}, \alpha_{\varphi}, \alpha_{\mathrm{z}}$ are the thermal expansion coefficients in the $\mathrm{r}, \varphi, \mathrm{z}$ directions [25], respectively.The stress equilibrium equation is written as,

$\int_{\Omega} \boldsymbol{B}^{T} \sigma d \Omega=\boldsymbol{F}$

where $\boldsymbol{B}$ is a shape function matrix inside an element, $\sigma$ denotes the stress vector, $\boldsymbol{F}$ is the external load vector. The stress-strain relation at $t+\Delta t$ is,

$\sigma^{t+\Delta t}=\boldsymbol{D}\left(\varepsilon^{t+\Delta t}-\varepsilon_{\mathrm{th}}^{t+\Delta t}-\varepsilon_{\mathrm{p}}^{t+\Delta t}\right)$

Since $\varepsilon_{\mathrm{p}}^{t+\Delta t}=\varepsilon_{\mathrm{p}}^{t}+\Delta \varepsilon_{\mathrm{p}}$, the stress equilibrium equation at $t+\Delta t$ can be written as,

$\int_{\Omega} \boldsymbol{B}^{T} \boldsymbol{D} \boldsymbol{\varepsilon}^{t+\Delta t} d \Omega=\int_{\Omega} \boldsymbol{B}^{T} \boldsymbol{D} \varepsilon_{t h}^{t+\Delta t} d \Omega+\int_{\Omega} \boldsymbol{B}^{T} \boldsymbol{D}\left(\varepsilon_{p}^{t}+\Delta \varepsilon_{p}\right) d \Omega+F^{t+\Delta t}$

where $\Delta \varepsilon_{p}=\dot{\varepsilon}_{\mathrm{p}}^{t} \cdot \Delta t$. When using the finite element method to solve the above equation, fixed displacement conditions are set at the top surface of the graphite layer, $u=w=0$. The symmetric condition is set at the axis, $u=0$. Free boundary conditions are set at other boundaries (Fig. 1).

\section{Results}

\subsection{Low-temperature and high-temperature plastic mechanisms}

In the following, we will obtain the mechanical parameters using the experimental data in the compression tests of silicon carbide crystals. Since there are two plastic deformation mechanisms, the slopes of high-temperature and low-temperature curves in Fig. 2 differ significantly. For constant-strain-rate compression tests, Eq. (4) is rewritten with replacement of $\tau_{\text {eff }}$ by $\tau_{\mathrm{CRSS}}$, as,

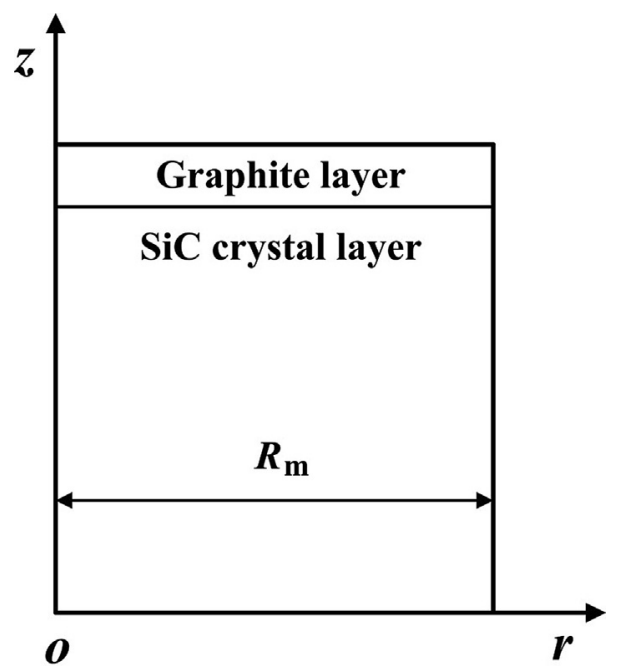

Fig. 1. Schematic of the coupled graphite/crystal layers.

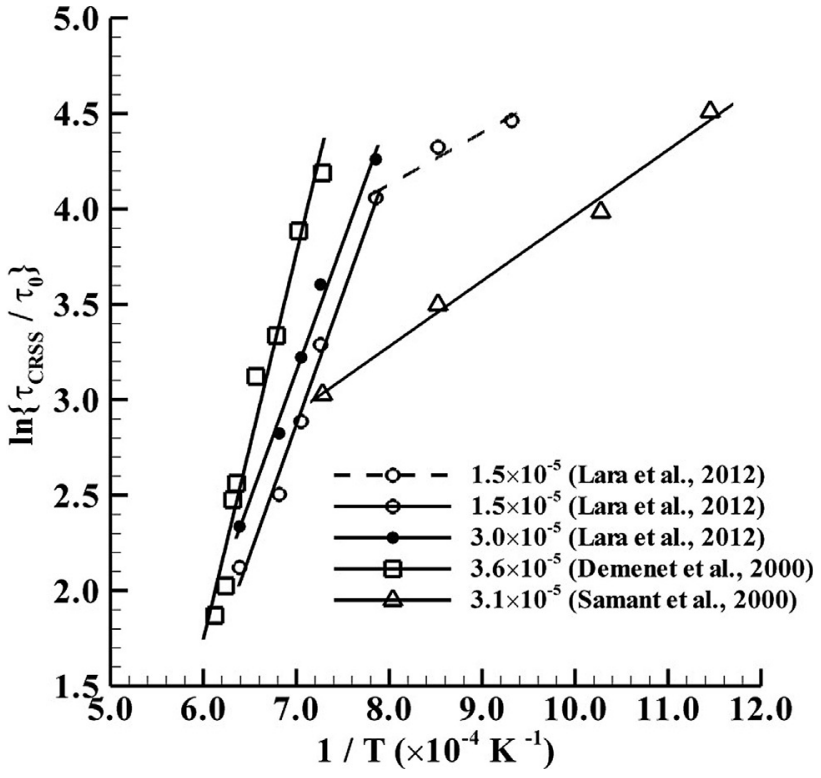

Fig. 2. Plot of $\ln \left\{\tau_{\mathrm{CRSS}} / \tau_{0}\right\}$ versus $1 / \mathrm{T}$ for the basal slip in $4 \mathrm{H}$-SiC at different strain rates. Symbols $\bigcirc$ and $\bigcirc$ represent experimental data from Ref. [14] and the strain rates $\dot{\varepsilon}$ are $1.5 \times 10^{-5} \mathrm{~s}^{-1}, 3.0 \times 10^{-5} \mathrm{~s}^{-1}$, respectively; symbol $\Delta$ represents experimental data from Ref. [27] for the strain rate of $3.1 \times 10^{-5} \mathrm{~s}^{-1}$ and $\square$ represents experimental data from Ref. [13] for the strain rate of $3.6 \times 10^{-5} \mathrm{~s}^{-1}$.

$\dot{\gamma}_{\mathrm{p}}=A\left(\frac{\tau_{\mathrm{CRSS}}}{\tau_{0}}\right)^{n} \exp \left(-\frac{Q}{k_{\mathrm{B}} T}\right)$

where $A$ is a constant [26]. The logarithmic form of the above equation is,

$\ln \left(\frac{\tau_{\mathrm{CRSS}}}{\tau_{0}}\right)=\frac{Q}{n k_{\mathrm{B}}} \frac{1}{T}+\frac{\ln \dot{\gamma}_{\mathrm{P}}-\ln A}{n}$

The linear relation between $\ln \left\{\tau_{\mathrm{CRSS}} / \tau_{0}\right\}$ and $1 / T$ is expected from the above equation if $Q / n$ remains constant (Fig. 2). Here, we can determine the relation between the activation energy and the stress exponent by the following equation,

$\frac{Q}{n}= \begin{cases}0.3 \mathrm{eV}, & T<T_{\text {trans }} \\ 1.3 \mathrm{eV}, & T>T_{\text {trans }}\end{cases}$

where $T_{\text {trans }}$ is the transition temperature.

Based on the relation between activation energy and stress exponent (Eq. (20)), we obtained the plastic parameters by fitting the calculated results to the experimental data at low and high temperatures (Table 1). The data in parenthesis in Table 1 are taken from Ref. [19]. The simulated stress-strain curves are plotted in Fig. 3. It clearly shows that the simulated results agree well with the measurements at both the low and high temperatures.

Table 1

Calculated plastic parameters for SiC crystals (Data in parenthesis are taken from [19]).

\begin{tabular}{llllll}
\hline$T\left({ }^{\circ} \mathrm{C}\right)$ & $Q(\mathrm{eV})$ & $n$ & $K \cdot v_{0}$ & $b \cdot v_{0}$ & $\lambda$ \\
\hline 800 & $0.9,(2.6)$ & $3.0(2.8)$ & $6.5 \times 10^{-10}$ & $9.9 \times 10^{-13}$ & $0.2(0.7)$ \\
900 & $0.9,(2.8)$ & $3.0(2.8)$ & $3.8 \times 10^{-10}$ & $6.0 \times 10^{-13}$ & $0.2(0.5)$ \\
1104 & $3.9,(3.3)$ & $3.0(2.8)$ & $1.0 \times 10^{+5}$ & $1.5 \times 10^{-2}$ & $0.8(1.0)$ \\
1145 & $3.9,(3.5)$ & $3.0(2.8)$ & $1.1 \times 10^{+5}$ & $1.3 \times 10^{-3}$ & $1(1.2)$ \\
1194 & $3.9,(3.2)$ & $3.0(2.8)$ & $3.4 \times 10^{+5}$ & $6.5 \times 10^{-3}$ & $1(1.1)$ \\
1292 & $3.9,(3.7)$ & $3.0(2.8)$ & $2.1 \times 10^{+5}$ & $6.6 \times 10^{-2}$ & $0.8(1.5)$ \\
\hline
\end{tabular}




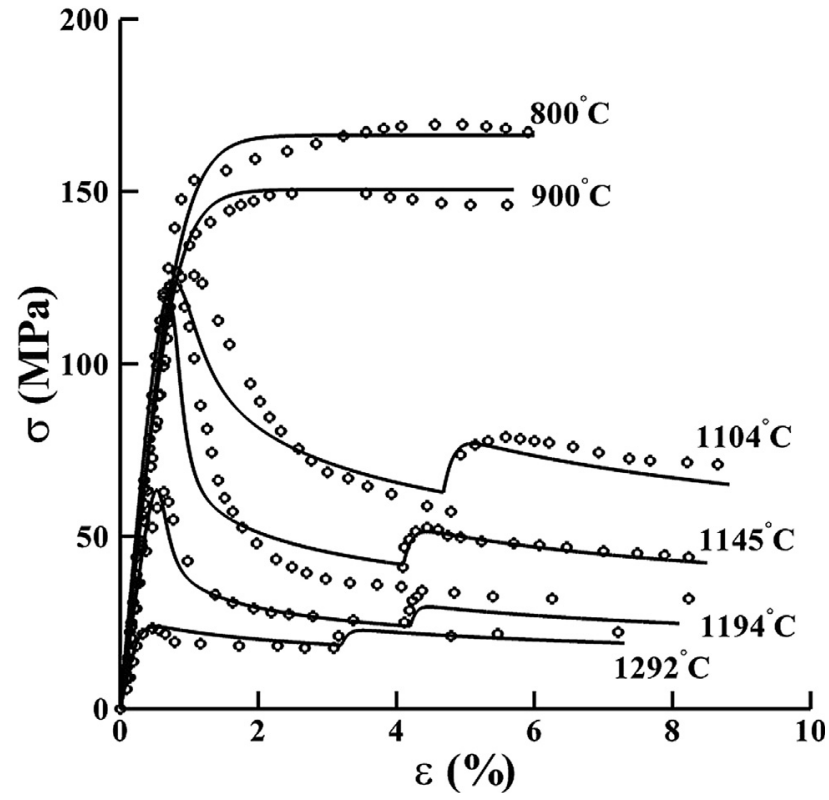

Fig. 3. Simulated stress(MPa)-versus-strain(\%) curves for $4 \mathrm{H}-\mathrm{SiC}$ at temperatures between 800 and $1300{ }^{\circ} \mathrm{C}$. The circles represent experimental data from Ref. [14]. The initial strain rate is $1.5 \times 10^{-5} \mathrm{~s}^{-1}$ for all tests. For tests at temperatures above $1000{ }^{\circ} \mathrm{C}$, the strain rate jumps to $3.0 \times 10^{-5} \mathrm{~s}^{-1}$ from the initail strain rate when the strain is close to $4 \%$.

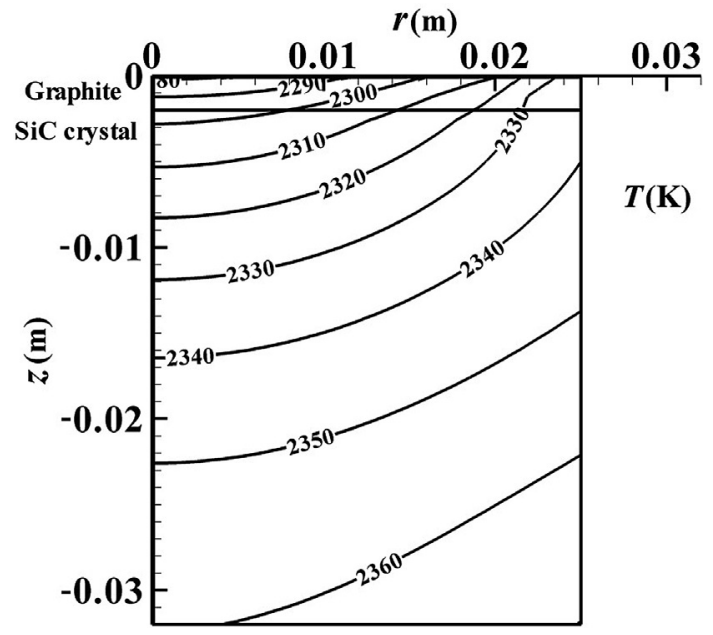

Fig. 4. Temperature distribution in the graphite and SiC crystal layers.

Table 2

Thermal conductivities of materials $(\mathrm{W} /(\mathrm{m} \cdot \mathrm{K}))$.

\begin{tabular}{lll}
\hline Temperature & Graphite & SiC crystal \\
\hline $2000{ }^{\circ} \mathrm{C}$ & 30.78 & 65.00 \\
$2250{ }^{\circ} \mathrm{C}$ & 28.80 & 60.00 \\
\hline
\end{tabular}

\subsection{Dislocation density in the growth process}

A typical physical vapor transport (PVT) growth system is considered here, where the radio frequency of $10 \mathrm{kHz}$ is used for induction heating. The thermal field in the graphite and $\mathrm{SiC}$ crystal layers is shown in Fig. 4, where the temperatures in graphite layer are lower than those in the crystal. The thermal conductivities of graphite and SiC crystal are listed in Table 2.

The calculated dislocation distribution which is caused by the thermal stress is shown in Fig. 5. It shows that the dislocation density

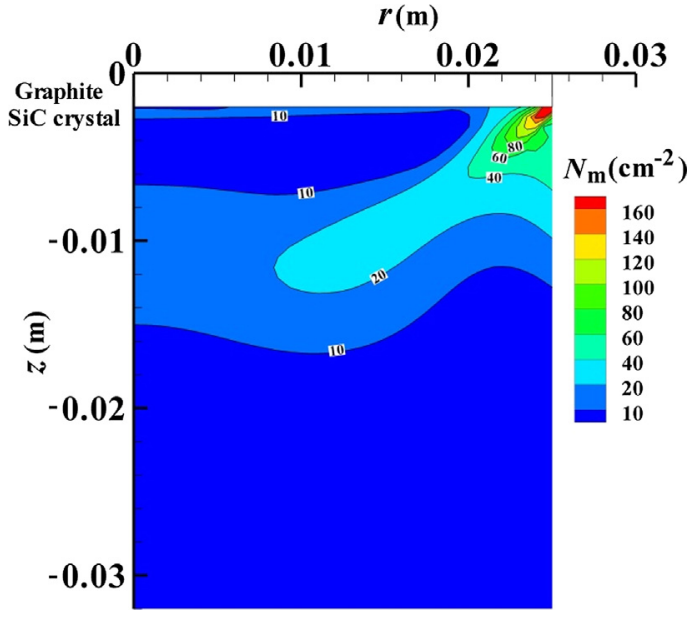

Fig. 5. Dislocation density distribution in the SiC crystal.

near the graphite/SiC interface is much higher than that in the bulk region. Large dislocation density is related to the temperature gradient near the graphite/SiC interface, which agrees with the experimental observations that the crystal quality improves with the crystal thickness. The maximum of the calculated dislocation densities in the crystal during the stable growth is around $240 \mathrm{~cm}^{-2}$.

As indicated in Ref. [28], the density of defects in the bulk SiC material can be obtained by dividing the total length of dislocations observed by the volume of the sample. According to Powell et al. [28], a high quality 4-degree off-axis $\mathrm{SiC}$ wafer having a diameter of at least 3 in. $(75 \mathrm{~mm}$ ) should contains at least one continuous square inch $\left(6.25 \mathrm{~cm}^{2}\right)$ of surface area having a basal plane dislocation density of less than about $500 \mathrm{~cm}^{-2}$. The calculation results for dislocation density in $4 \mathrm{H}-\mathrm{SiC}$ crystals agree qualitatively with the experimental data.

In order to explore the dislocatin evolution during the growth process, we selected six points to detect the changes. At the graphite/ crystal interface, points $1,2,3$ with $\mathrm{r}=0,0.0125,0.025 \mathrm{~m}$ and $\mathrm{z}=-0.002 \mathrm{~m}$ are selected. In the middle part of crystal, points $4,5,6$ with $\mathrm{r}=0,0.0125,0.025 \mathrm{~m}$ and $\mathrm{z}=-0.017 \mathrm{~m}$ are selected. The evolutions of the dislcoation and the Von Mises stress are shown in Fig. 6 and Fig. 7, respectively. The Von Mises stress is defined as,

$\sigma_{\text {Mises }}=\sqrt{\frac{1}{2}\left[\left(\sigma_{z z}-\sigma_{r r}\right)^{2}+\left(\sigma_{z z}-\sigma_{\varphi \varphi}\right)^{2}+\left(\sigma_{\varphi \varphi}-\sigma_{r r}\right)^{2}+6 \tau_{r z}^{2}\right]}$

In Fig. 6a, the Von Mises stress drasticly changes within $t=0.1 \mathrm{~s}$ because of the strong coupling of the dislcoation and stresses. At $t=100 \mathrm{~s}$, the dislocation stresses are in equilibrium with the applied stresses. This implys that the dislocatin evolution is much faster than crystal growth rate. The evolution of dislocation can be treated to be reasonable during the growth stage. The dislocation density near the edge of the seed is much higher than that in the central region of the seed. Similarly, the stresses at points 4, 5, 6 decrease rapidly (Fig. 7a).

\section{Conclusions}

The plastic deformation behaviors of $4 \mathrm{H}-\mathrm{SiC}$ single crystals are studied. We first predict the 1-D stress-strain relationship of the SiC material using the Alexander-Haasen $(\mathrm{AH})$ model when temperature is in the range of 800 to $1300{ }^{\circ} \mathrm{C}$. Low-temperature and high-temperature plastic mechanisms are analyzed and plastic parameters are obtained. The ratio of the activation energy and stress exponent is set as 0.3 for temperatures lower than the transition temperature, and $1.3 \mathrm{eV}$ for temperatures higher than the transition temperature.

The AH model is then used to simulate the dislocation density distribution in the SiC crystal grown by the PVT method. It's found that large dislocation occurs near the graphite/SiC interface where the 


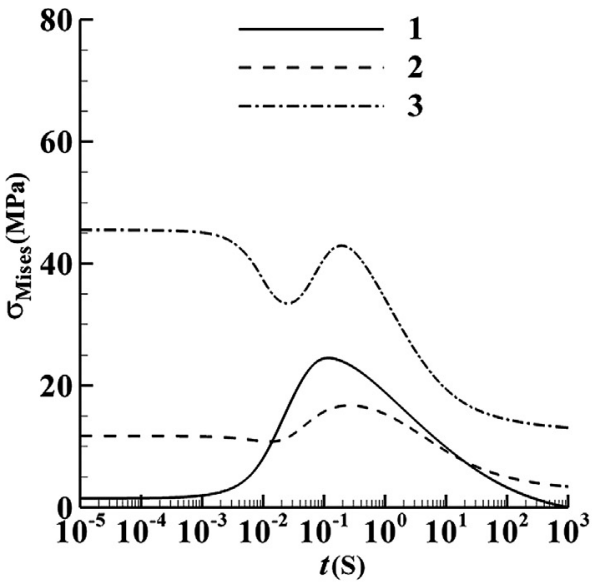

(a)

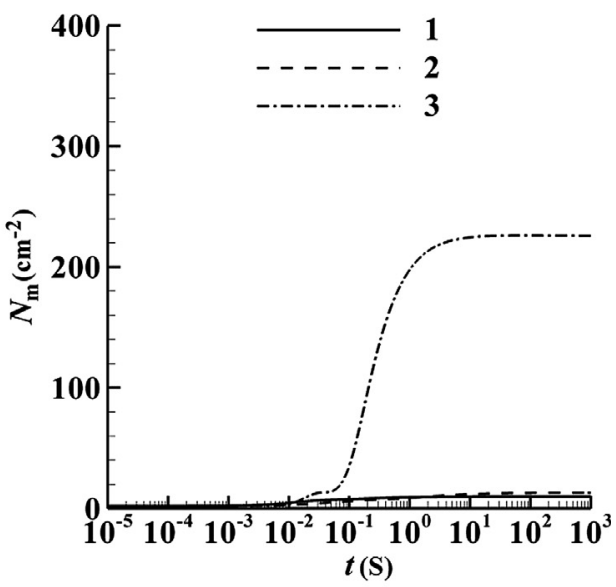

(b)

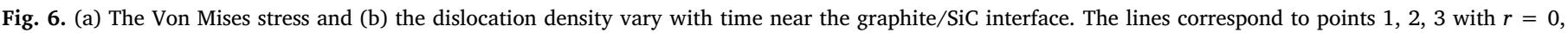
$0.0125,0.025 \mathrm{~m}$ and $z=-0.002 \mathrm{~m}$.

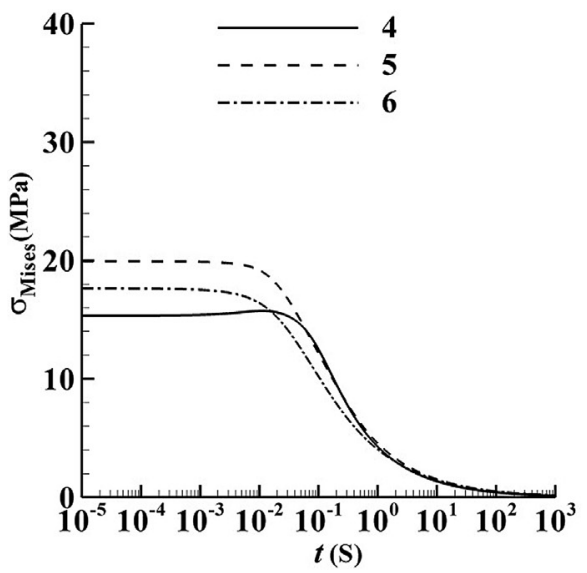

(a)

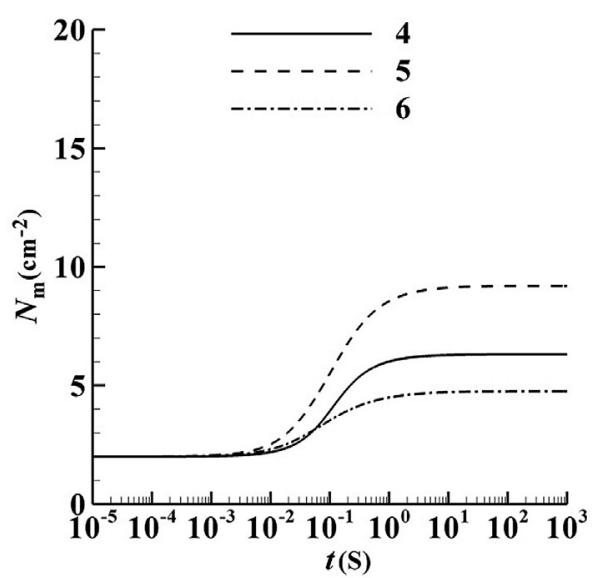

(b)

Fig. 7. (a) The Von Mises stress and (b) the dislocation density vary with time in the central region of the crystal, the lines correspond to points $4,5,6$ with $r=0$, $0.0125,0.025 \mathrm{~m}$ and $z=-0.017 \mathrm{~m}$.

temperature gradient is large. The maximum of the calculated dislocation densities is around $240 \mathrm{~cm}^{-2}$, which qualitatively agrees with the experimental data. The plastic model can be used to improve the understanding of plastic deformation of $\mathrm{SiC}$ crystals and to improve the $\mathrm{SiC}$ crystal growth process.

\section{Author contribution}

Q.-S. Chen developed the idea for the study, P. Zhu and M. He did the analyses. All authors were involved in writing the manuscript.

\section{Declaration of Competing Interest}

The authors declare that they have no known competing financial interests or personal relationships that could have appeared to influence the work reported in this paper.

\section{Acknowledgments}

This work has been supported by the National Natural Science Foundation of China (11772344, 11532015).

\section{References}

[1] P.J. Wellmann, Review of SiC crystal growth technology, Semicond. Sci. Technol. 33 (2018) 103001.

[2] M.S. Ramm, E.N. Mokhov, S.E. Demina, M.G. Ramm, A.D. Roenkov, YuA. Vodakov, A.S. Segal, A.N. Vorob'ev, S.Yu Karpov, A.V. Kulik, YuN. Makarov, Optimization of sublimation growth of SiC bulk crystals using modeling, Mater. Sci. Eng. B61-62 (1999) 107-112.

[3] M. Selder, L. Kadinski, Yu. Makarov, F. Durst, P. Wellmann, T. Straubinger, D. Hofmann, S. Karpov, M. Ramm, Global numerical simulation of heat and mass transfer for SiC bulk crystal growth by PVT, J. Cryst. Growth 211 (2000) 333-338.

[4] O.-S. Chen, H. Zhang, V. Prasad, C.M. Balkas, N.K. Yushin, Modeling of heat transfer and kinetics of physical vapor transport growth of silicon carbide crystals, J. Heat Trans. 123 (2001) 1098-1109.

[5] J. Geiser, O. Klein, P. Philip, Numerical simulation of temperature fields during the sublimation growth of SiC single crystals using WIAS-HiTNIHS, J. Cryst. Growth 303 (2007) 352-356.

[6] D. Klimm, Electronic materials with a wide band gap: recent developments, IUCrJ 1 (2014) 281-290.

[7] I.D. Matukov, D.S. Kalinin, M.V. Bogdanov, S.Yu. Karpov, D.Kh. Ofengeim, M.S. Ramm, J.S. Barash, E.N. Mokhov, A.D. Roenkov, Yu.A. Vodakov, M.G. Ramm, H. Helava, Yu.N. Makarov, Modeling of facet formation in SiC bulk crystal growth, J. Cryst. Growth 266 (2004) 313-319.

[8] E. Schmitt, T. Straubinger, M. Rasp, A.-D. Weber, Defect reduction in sublimation grown SiC bulk crystals, Superlattice Microst. 40 (2006) 320-327.

[9] G. St, R.C. Müller, H.M. Glass, V.F. Hobgood, M. Tsvetkov, D. Brady, J.R. Henshall, D. Jenny, C.H. Carter Malta, Jr., The status of SiC bulk growth from an industrial point of view, J. Cryst. Growth 211 (2000) 325-332.

[10] J.Q. Liu, M. Skowronski, C. Hallin, R. Söderholm, H. Lendenmann, Structure of recombination-induced stacking faults in high-voltage SiC p-n junctions, Appl. Phys. Lett. 80 (2002) 749-751. 
[11] S. Fujita, K. Maeda, S. Hyodo, Dislocation glide motion in 6H SiC single crystals subjected to high-temperature deformation, Phil. Mag. A 55 (1987) 203-215.

[12] A.V. Samant, P. Pirouz, Activation parameters for dislocation glide in $\alpha$-SiC, Int. J. Refract. Metals Hard Mater. 16 (1998) 277-289.

[13] J.-L. Demenet, M.H. Hong, P. Pirouz, Plastic behavior of 4H-SiC single crystals deformed at low strain rates, Scrip. Mater. 43 (2000) 865-870.

[14] A. Lara, A. Muñoz, M. Castillo-Rodríguez, A. Domínguez-Rodríguez, Plastic behavior of $4 \mathrm{H}$-SiC single crystals deformed at temperatures between 800 and $1300^{\circ} \mathrm{C}$ Ceramics Int. 38 (2012) 1381-1390.

[15] K. Sumino, I. Yonenaga, Dislocation dynamics and mechanical behavior of elemental and compound semiconductors, Phys. Stat. Sol. 138 (1993) 573-581.

[16] N. Miyazaki, H. Uchida, T. Munakata, K. Fujioka, Y. Sugino, Thermal stress analysis of silicon bulk single crystal during Czochralski growth, J. Cryst. Growth 125 (1992) 102-111.

[17] J. Völkl, G. Müller, A new model for the calculation of dislocation formation in semiconductor melt growth by taking into accout the dynamics of plastic deformation, J. Cryst. Growth 97 (1989) 136-145.

[18] S.Yu. Karpov, A.V. Kulik, M.S. Ramm, Yu.N. Makarov, Dynamics of 4H-SiC plasti city, Mater. Sci. Forum 433-436 (2003) 297-300.

[19] B. Gao, K. Kakimoto, Dislocation-density-based modeling of the plastic behavior of 4H-SiC single crystals using the Alexander-Haasen model, J. Cryst. Growth 386
(2014) 215-219.

[20] J. Cochard, I. Yonenaga, S. Gouttebroze, M. M'Hamdi, Z.L. Zhang, Constitutive modeling of intrinsic silicon monocrystals in easy glide, J. Appl. Phys. 107 (2010) 033512 .

[21] E. Orowan, Problems of plastic gliding, Proc. Phys. Soc. 52 (1940) 8-22.

[22] U. Messerschmidt, Dislocation Dynamics During Plastic Deformation, Springer, Berlin, 2010.

[23] W.-W. Xu, F. Xia, L. Chen, M. Wu, T. Gang, Y. Huang, High-temperature mechanical and thermodynamic properties of silicon carbide polytypes, J. Alloys Compd. 768 (2018) 722-732.

[24] O.W. Dillon Jr., C.T. Tsai, R.J. DeAngelis, Dislocation dynamics of web type silicon ribbon, J. Cryst. Growth 82 (1987) 50-59.

[25] K. Kamitani, M. Grimsditch, J.C. Nipko, C.-K. Loong, M. Okada, I. Kimura, The elastic constants of silicon carbide: a Brillouin-scattering study of $4 \mathrm{H}$ and $6 \mathrm{H} \mathrm{SiC}$ single crystals, J. Appl. Phys. 82 (1997) 3152-3154.

[26] H. Alexander, Dislocations in covalent crystals, in: F.R.N. Nabarro (Ed.), Dislocations in Solids, Elsevier North-Holland, Amsterdam, 1986, pp. 113-234.

[27] A.V. Samant, M.H. Hong, P. Pirouz, The relationship between activation parameters and dislocation glide in 4H-SiC single crystals, Phys. Stat. Sol. B 222 (2000) 75-93.

[28] A. Powell, M. Brady, V.F. Tsvetkov, Low basal plane dislocation bulk grown SiC wafers, U.S. Patent No. 20060075958, 2006. 